\title{
Classification of gamma-ray bursts observed by INTEGRAL
}

\author{
A. Martin-Carrillo*1, M. Topinka ${ }^{1}$, L. Hanlon ${ }^{1}$, S. Meehan ${ }^{1}$, S. Foley ${ }^{1,2}$, B. McBreen ${ }^{1}$ \\ ${ }^{1}$ UCD School of Physics, Dublin, Ireland \\ ${ }^{2}$ Max Planck Institut Garching (MPE) \\ E-mail: antonio.martin-carrillo@ucd.ie
}

Long gamma-ray bursts (GRBs) can be divided into three classes ('classical' GRBs, X-ray rich bursts and X-ray flashes), based on the ratio of their fluences in a soft energy band to a hard energy band. However, the other characteristics of the X-ray rich bursts and X-ray flashes resemble those of the classical GRBs, suggesting that they are closely related phenomena. We present the classification of more than 70 GRBs triggered by the IBAS alert on IBIS/ISGRI since the launch of INTEGRAL's. An optimised criterion for the classification has been carried out, based on the 20-50 keV and 50-200 keV energy bands. A comparison between the distributions of each burst type found in the INTEGRAL sample, and those identified by Swift and HETE-2, is also presented.

8th INTEGRAL Workshop "The Restless Gamma-ray Universe"- Integral2010,

September 27-30, 2010

Dublin Ireland

* Speaker. 


\section{Introduction}

The study of the prompt emission from gamma-ray bursts (GRBs) can provide important insights into the mechanisms whereby the high-energy radiation is produced in these extremely powerful events. GRB emission in the prompt phase is generally interpreted as synchrotron radiation from high-energy electrons that are accelerated in internal shocks [1]. In the last decade, the increased capabilities of missions such as HETE-2 [2]; Swift [3]; INTEGRAL [4, 5] and, most recently, Fermi $[6,7]$ have led to much better locations, greater sensitivity to faint bursts, improvements in the determination of temporal and spectral properties and rapid multi-wavelength follow-up observations, leading to substantial development in our understanding of GRBs [8,9].

This increase in detection capability has led to the discovery of two new groups of bursts with similar properties to the 'classical' GRBs, but with much softer spectra, emitting most of their energy in the X-ray band. These bursts are referred to as X-ray flashes (XRFs) and X-ray rich (XRR) bursts $[10,11]$. The three classes of bursts seem closely related to each other, but the exact nature of their connection remains unclear. For example, it has been suggested that the observed spectral differences are the result of variations in observer viewing angle relative to the jet axis [12]. This work presents a robust classification scheme for the different types of burst in the INTEGRAL data that will allow a future detailed comparison of their spectral and temporal characteristics, the detailed study of which is a necessary first step towards to a better understanding of relationships between bursts.

\section{GRB sample}

Since its launch in October 2002, INTEGRAL has triggered 78 GRBs with the INTEGRAL Burst Alert System (IBAS) using the IBIS detector [13] in the 20-200 keV energy range. INTE$G R A L$ is not a mission designed specifically to detect GRBs but, thanks to the sensitivity and wide field of view $\left(29^{\circ} \times 29^{\circ}\right.$ down zero response) of the IBIS detector, about $0.8 \mathrm{GRBs} / \mathrm{month}$ are detected. The spatial distribution of all bursts detected up to September 2010 is shown in Fig 1.

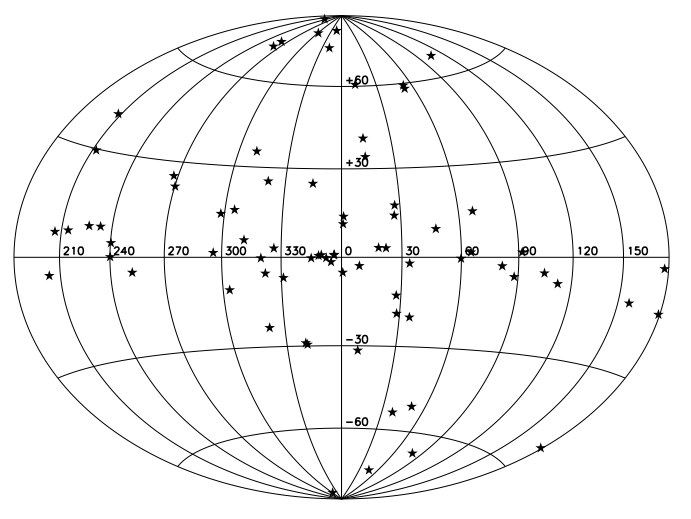

Figure 1: Spatial distribution of all GRBs triggered by IBIS/ISGRI, in galactic coordinates. The non uniform distribution of bursts reflects the pointing observations of the detector. 
A limited sample was used in this study consisting of 49 GRBs detected by IBIS/ISGRI, corresponding to all the events that can be analysed using the standard OSA 9 procedure (bright GRBs in the 20-200 keV energy range with duration longer than $1 \mathrm{~s}$ and all the prompt emission observed in pointing mode). A full analysis of the complete INTEGRAL GRB sample will be presented by A. Martin-Carrillo et al. (2011). JEM-X detected 20 of the 49 GRBs in the sample, for which the off-axis angle was less than $5^{\circ}$. For these bursts, the extended energy range from $3 \mathrm{keV}$ to $1 \mathrm{MeV}$ is used in the analysis.

\section{GRBs, XRRs and XRFs in the INTEGRAL sample}

The classification of bursts as GRBs, XRRs or XRFs, based on the ratio of their fluences in different energy ranges, has been carried out for HETE-2 [2] and Swift [3]. The energy ranges were optimised to the corresponding energy sensitivities of the instruments. In the Swift sample, the burst subclasses were based on the fluence ratio in the $25-50 \mathrm{keV}$ and $50-100 \mathrm{keV}$ bands, while for HETE-2 the classification was based on the fluence ratio in the $2-30 \mathrm{keV}$ and $30-400 \mathrm{keV}$ energy ranges. For the INTEGRAL sample, the softer energy bands can only be used for the 20 bursts for which JEM-X data is available.

Based on the sensitivity of the IBIS/ISGRI detector, the optimum energy bands for the INTEGRAL sample are 20-50 keV and 50-200 keV. In order to make a definition for the classification of GRBs using the fluence ratio $\mathrm{S}(20-50 \mathrm{keV}) / \mathrm{S}(50-200 \mathrm{keV})$ that is compatible with previous work, the fluence ratio of a theoretical burst was determined using the Band model [14] with spectral parameters $\alpha=-1, \beta=-2.5$. These values of $\alpha$ and $\beta$ are typical of GRBs detected by BATSE $[15,16]$ and HETE-2 [2]. $\mathrm{E}_{\text {peak }}$ values were chosen to cover the range between XRFs and XRRs $\left(\mathrm{E}_{\text {peak }}=\right.$ $30 \mathrm{keV})$ and $\mathrm{XRRs}$ and GRBs $\left(\mathrm{E}_{\text {peak }}=100 \mathrm{keV}\right)$ [2]. Fig 2 shows the ratio $\mathrm{S}(20-50 \mathrm{keV}) / \mathrm{S}(50-$ $200 \mathrm{keV}$ ) versus the ratio $\mathrm{S}(25-50 \mathrm{keV}) / \mathrm{S}(50-100 \mathrm{keV})$ used by Swift [3] for the INTEGRAL GRB sample. All the classifications remain unchanged in the transformation from the Swift energy bands to the INTEGRAL energy bands.

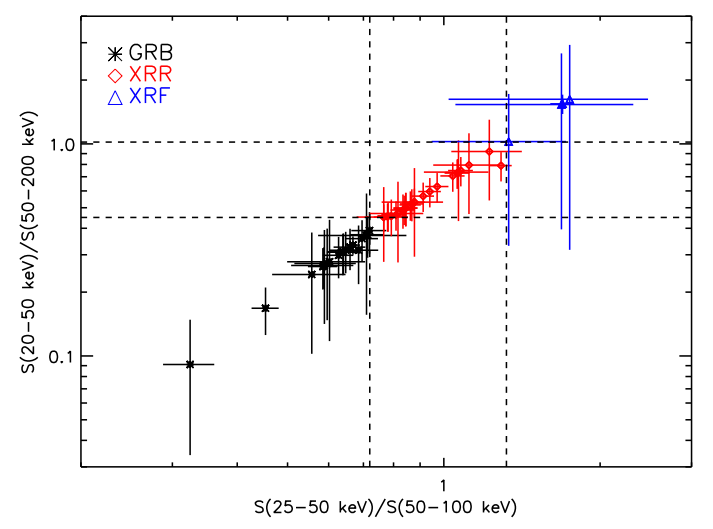

Figure 2: Comparison of fluence ratios using the IBIS/ISGRI (S(20-50 keV)/S(50-200 keV) and the Swift $(\mathrm{S}(25-50 \mathrm{keV}) / \mathrm{S}(50-100 \mathrm{keV})$ energy bands over the INTEGRAL GRB sample. The dashed lines represent the boundaries between each class of burst. 
On the basis of this analysis, bursts in the INTEGRAL sample can be classified according to the following criteria:

$$
\begin{gathered}
\mathrm{S}(20-50) / \mathrm{S}(50-200) \leq 0.45 \text { for GRBs } \\
0.45<\mathrm{S}(20-50) / \mathrm{S}(50-200) \leq 1.02 \text { for XRR bursts } \\
\mathrm{S}(20-50) / \mathrm{S}(50-200)>1.02 \text { for XRFs }
\end{gathered}
$$

Table 1 presents the classification of the INTEGRAL bursts based on these fluence ratios.

Table 1: List of 49 IBIS/ISGRI bursts in each of the 3 categories

\begin{tabular}{cc|ccc|c}
\hline \multicolumn{2}{c}{ GRB } & \multicolumn{3}{c}{ XRR } & XRF \\
\hline \hline 030320 & 060901 & 030227 & 050918 & 080120 & 040812 \\
031203 & 061025 & 030501 & $050922 \mathrm{~A}$ & 080414 & 040903 \\
041015 & 070311 & 040223 & $051105 \mathrm{~B}$ & 080922 & 060130 \\
041218 & 070615 & 040403 & $060204 \mathrm{~A}$ & $081003 \mathrm{~A}$ & 091202 \\
041219A & 070925 & 040730 & $060912 \mathrm{~B}$ & $081003 \mathrm{~B}$ & \\
050502A & 071109 & 040827 & 060930 & 081204 & \\
050504 & 080613 & 050626 & 061122 & 091015 & \\
050520 & $080723 \mathrm{~B}$ & $050714 \mathrm{~A}$ & 070309 & $100331 \mathrm{~A}$ & \\
050522 & 081016 & & & $100518 \mathrm{~A}$ & \\
060114 & 091111 & & & & \\
\hline \hline
\end{tabular}

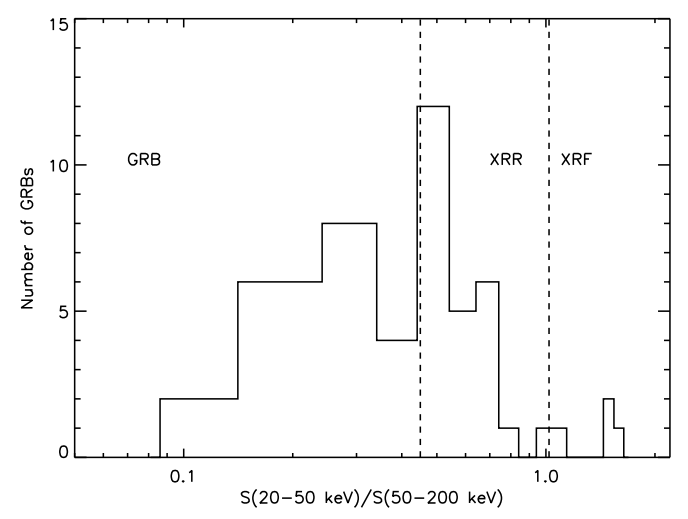

Figure 3: Histogram of the fluence ratio $\mathrm{S}(20-50 \mathrm{keV}) / \mathrm{S}(50-100 \mathrm{keV})$ for IBIS/ISGRI bursts listed in Table 1 . The dashed lines represent the boundaries between each class.

\section{Discussion}

In the INTEGRAL sample, $8 \%$ of bursts are classified as XRFs, $51 \%$ as XRRs and $41 \%$ as GRBs (Fig. 3). These results are broadly in agreement with the larger sample from Swift [3]. 
From the sample of 158 Swift GRBs, 6\% were classified as XRFs, 61\% as XRRs and $33 \%$ as GRBs. The agreement shown in Fig. 2 between the classification using Swift and INTEGRAL criteria shows the consistency of the new boundary limits. A comparison between the HETE-2 bands $(\mathrm{S}(2-30 \mathrm{keV}) / \mathrm{S}(30-400 \mathrm{keV}))$ and the IBIS/ISGRI bands, using the 20 bursts for which JEM$\mathrm{X}$ data is available also yields consistent results (A. Martin-Carrillo et al. (2011) in preparation).

In Fig. 4, the distribution of XRFs, XRRs and GRBs in the S(50-200 keV) vs $S(20-50 \mathrm{keV})$ plane is shown. A linear correlation is clearly visible, with a best fit of:

$$
\mathrm{S}(50-200 \mathrm{keV})=(0.55 \pm 0.41) \times \mathrm{S}(20-50 \mathrm{keV})^{(1.03 \pm 0.05)}
$$

This slope is not as steep as that obtained with the HETE-2 sample (power-law slope $=1.28 \pm 0.08$ ) which may be caused by the different sensitivity of HETE-2 to different burst types compared to INTEGRAL [2].

The duration distributions of the different classes is quite wide with median values of $\sim 35 \mathrm{~s}$ and $\sim 20 \mathrm{~s}$ for GRBs and XRRs bursts respectively. No median value has been estimated for XRFs due to the lack of bursts in that category. Thus, a clear distinction between them based on their $\mathrm{T}_{90}$ is not possible.

Future work will incorporate the latest GRBs triggered by IBIS/ISGRI in the sample and on the study of the range of spectral parameters for each category (A. Martin-Carrillo et al. (2011) in preparation).

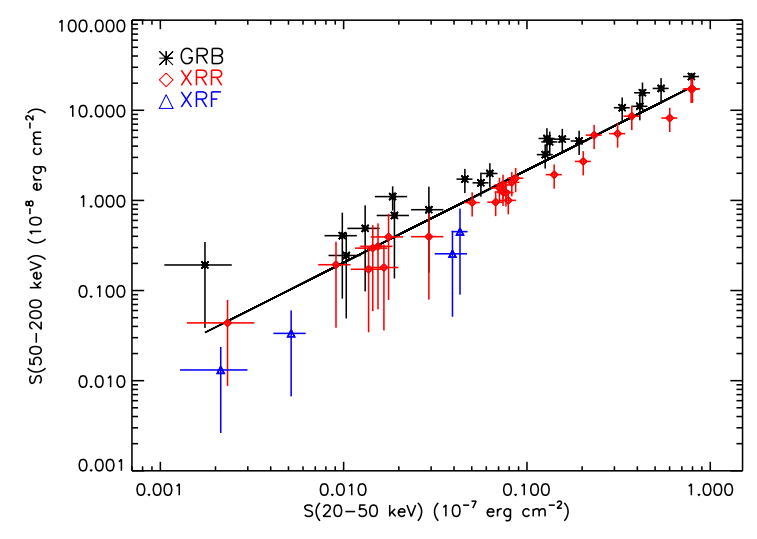

Figure 4: The GRB fluences on $20-50 \mathrm{keV}$ and $50-200 \mathrm{keV}$ for the 49 bursts considered in this study. The solid line represents the best fit power-law to the data.

\section{Acknowledgements}

This study is based on observations with INTEGRAL, an ESA project with instruments and science data centre funded by ESA member states (especially the PI countries: Denmark, France, Germany, Italy, Switzerland, Spain), Poland and with the participation of Russia and the USA. AMC and LH wish to acknowledge financial support from SFI grant 09/RFP/AST/2400. 


\section{References}

[1] Daigne, F.; Bosnjak, Z; Dubus, G., 2011 A\&A 526, 110

[2] Sakamoto, T., Lamb, D. Q., Kawai, N., Yoshida, A. et al. 2005 ApJ 629, 311

[3] Sakamoto, T., Hullinger, D., Sato, G. et al. 2008 ApJ 679, 570

[4] Foley, S., McGlynn, S., Hanlon, L. et al. 2008 A\&A 484, 143

[5] Vianello, G., Goetz, D., Mereghetti, S. 2009 A\&A 495, 1005

[6] Paciesas W. S. and Fermi Collaboration 2010 HEAD 11, Vol 41, 669

[7] Bissaldi, E.; von Kienlin, A.; Kouveliotou, C. et al. 2011 astro-ph/1101.3325

[8] Meszaros, P. 2006 RPPh 69, 2259

[9] Zhang, B. 2011 astro-ph/1104.0932v1

[10] Heise, J.; in’t Zand, J.; Kippen, R. M.; Woods, P. M. 2001 Proc. Second Rome Workshop: GRBs in the Afterglow Era p. 16, astro-ph/0111246v1

[11] Barraud, C.; Daigne, F.; Mochkovitch, R.; Atteia, J. L. 2005 A\&A 440, 809

[12] Lamb, D. Q.; Donaghy, T. Q. and Graziani, C. 2005 ApJ 620, 355

[13] Ubertini, P.; Lebrun, F.; Di Cocco, G. et al. 2003 A\&A 411, L131

[14] Band, D.; Matteson, J.; Ford, L. et. al 1993 ApJ 413, 281

[15] Preece, R. D., Briggs, M. S., Mallozzi, R. S. et al. 2000 ApJS 126, 19

[16] Kaneko, Y., Preece, R. D., Briggs, M. S. et al. 2006 ApJS 166, 298 